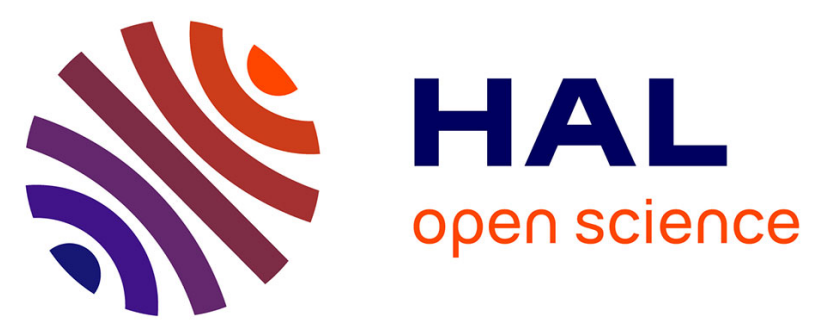

\title{
Brain networks of rats under anesthesia using resting-state fMRI: comparison with dead rats, random noise and generative models of networks
}

Guillaume Jean-Paul Claude Becq, Emmanuel Luc Barbier, Sophie Achard

\section{- To cite this version:}

Guillaume Jean-Paul Claude Becq, Emmanuel Luc Barbier, Sophie Achard. Brain networks of rats under anesthesia using resting-state fMRI: comparison with dead rats, random noise and generative models of networks. Journal of Neural Engineering, 2020, 17 (4), pp.045012. 10.1088/1741-2552/ab9fec . hal-02935391

\section{HAL Id: hal-02935391 \\ https://hal.science/hal-02935391}

Submitted on 10 Sep 2020

HAL is a multi-disciplinary open access archive for the deposit and dissemination of scientific research documents, whether they are published or not. The documents may come from teaching and research institutions in France or abroad, or from public or private research centers.
L'archive ouverte pluridisciplinaire $\mathbf{H A L}$, est destinée au dépôt et à la diffusion de documents scientifiques de niveau recherche, publiés ou non, émanant des établissements d'enseignement et de recherche français ou étrangers, des laboratoires publics ou privés. 


\title{
Brain networks of rats under anesthesia using resting-state fMRI: comparison with dead rats, random noise and generative models of networks
}

\author{
G. J.-P. C. Becq ${ }^{1}$, E. L. Barbier ${ }^{2,3}$, S. Achard ${ }^{1,4}$ \\ 1. Univ. Grenoble Alpes, CNRS, Grenoble INP, Gipsa-lab, 38000, Grenoble, France \\ 2. Univ. Grenoble Alpes, Inserm, U1216, Grenoble Institut Neurosciences, GIN, \\ 38000 Grenoble, France \\ 3. Univ. Grenoble Alpes, Inserm, UMS017, CNRS, US3552, CHU Grenoble Alpes, \\ IRMaGe, 38000 Grenoble, France \\ 4. Univ. Grenoble Alpes, CNRS, Inria, Grenoble INP, LJK, 38000 Grenoble, France \\ E-mail: guillaume.becq@grenoble-inp.fr, \\ sophie.achard@univ-grenoble-alpes.fr \\ emmanuel.barbier@univ-grenoble-alpes.fr
}

Sept 2019

\begin{abstract}
Objective Connectivity networks are crucial to understand the brain resting-state activity using functional magnetic resonance imaging (rs-fMRI). Alterations of these brain networks may highlight important findings concerning the resilience of the brain to different disorders. The focus of this paper is to evaluate the robustness of brain network estimations, discriminate them under anesthesia and compare them to generative models. Approach The extraction of brain functional connectivity (FC) networks is difficult and biased due to the properties of the data: low signal to noise ratio, high dimension low sample size. We propose to use wavelet correlations to assess FC between brain areas under anesthesia using four anesthetics (isoflurane, etomidate, medetomidine, urethane). The networks are then deduced from the functional connectivity matrices by applying statistical thresholds computed using the number of samples at a given scale of wavelet decomposition. Graph measures are extracted and extensive comparisons with generative models of structured networks are conducted. Main results The sample size and filtering are critical to obtain significant correlations values and thereby detect connections between regions. This is necessary to construct networks different from random ones as shown using rs-fMRI brain networks of dead rats. Brain networks under anesthesia on rats have topological features that are mixing small-world, scale-free and random networks. Betweenness centrality indicates that hubs are present in brain networks obtained from anesthetized rats but locations of these hubs are altered by anesthesia. Significance Understanding the effects of anesthesia on brain areas is of particular importance in the context of animal research since animal models are commonly used to explore functions, evaluate lesions or illnesses, and test new drugs. More generally, results indicate that the use of correlations in the context of fMRI signals is robust but must be treated with caution. Solutions are proposed in order to control spurious correlations by setting them to zero.
\end{abstract}


Keywords: resting-state functional magnetic resonance imaging (rs-fMRI), rats, anesthesia, brain connectivity networks, wavelets, generative models of networks. Submitted to: J. Neural Eng.

\section{Introduction}

Modeling the brain as a complex network has attracted a lot of interest in recent years. Structural networks, also called connectomes, are constructed for modeling the anatomical structure of the brain [1], while functional networks are extracted when observing the brain in operation [2]. In general, a node of the network corresponds to a brain region and an edge corresponds to a structural or a functional connection. The identification of connectomes or functional brain connectivity networks is challenging and several approaches have been proposed [3]. Once the networks have been obtained, they can be compared to generative models [4] or processed for classification [5]. The small-world model has been introduced as a model for human brain networks derived from diffusion tensor imaging (DTI) or functional magnetic resonance imaging (fMRI) data [6]. In particular, the small-world model ensures a balance between low mean shortest path and high clustering [7]. However, spurious detections of small-world networks may be obtained due to the sparsity of the observed networks [8]. A graph is said to be sparse when the number of edges present in the graph is much smaller than the total number of possible edges in the graph. Most of the time the observed networks from different research fields are sparse [9]. However the connectome of the monkey brain was found to be dense and not small-world using an invasive technique [10]. This may seem to contradict previous results and argue for new methodologies to assess the robustness of human brain networks extraction [11].

The identification of functional brain networks is complex, and many articles have already reported that spurious behaviours can be observed when data processing is not correct. Measures of correlations or partial correlations are biased by outliers [12, 13 , 14]. Sample size is a crucial parameter for accurate results [15]. Indeed, when the sample size is too small, the estimated correlations of Gaussian noise have high values. This is the result of the combination of two statistical problems, multiple comparisons and finite observations with a given number of samples [16, 8]. These two problems affect the identification of the graph edges and can have dramatic effects on the extraction of the graph measurements [17, 18].

In this paper, we challenge the methodologies for brain networks construction and the analyses using both experimental data sets and simulated networks. More specifically, pairwise correlations of signals are retained to evaluate functional connectivity using wavelets and to compute graph measures. It is worth mentioning that other methods exist to evaluate functional connectivity using rs-fMRI [19]. However, to the best of our knowledge, filtering with dyadic wavelets is the only filtering method 
where theoretical results are available to control the extraction of significant correlations. Numerical comparisons between methods to evaluate functional connectivity using rsfMRI is out of the scope of this study. The experimental data are brain signals of anesthetized or dead rats recorded during resting-state fMRI (rs-fMRI) sessions. First, we show that the choice of the threshold to identify the edges of the graphs can be controlled using Gaussian noise. This Gaussian noise is perfectly matching real data sets from dead rats. The graphs obtained for different anesthetics are then compared to generative models of networks obtained by the simulation of various functional networks with different topological properties. Finally, graph measures are extracted for each brain region and each anesthetic in order to localise the effect of the anesthetic on the brain maps.

\section{Materials and Methods}

\subsection{Description of the experimental protocol}

2.1.1. Animals All experiments were approved by the local ethics committee and were performed in full compliance with the guidelines of the European community (EUVD 86/609/EEC) for the care and use of the laboratory animals, under permits from the French Ministry of Agriculture (number 380820 for EB and B3851610008 for experimental and animal care facilities). Experiments were conducted in the animal research facility of the Grenoble Institute of Neurosciences, a Specific Pathogen Free (SPF) housing facility. Male rats were stabulated in enriched cages, with 3 animals per cage, with access to food and water ad libitum. The acquisition campaigns for each anesthetic were carried out one after the other, with animals ascribed to the group upon arrival order, without further randomization. Once all anesthestics were tested, about half of the data were obtained. A second run of acquisition campaigns was then performed to obtain the full dataset.

2.1.2. Experimental protocol Four anesthetics were evaluated in Long Evans rats (with $\mathrm{n}$ rats in each group): Etomidate (Eto- $\mathrm{L}, \mathrm{n}=7$ ), Isoflurane (Iso- $\mathrm{L}, \mathrm{n}=6$ ), Medetomidine (Med-L, $\mathrm{n}=7$ ) and Urethane (Ure-L, $\mathrm{n}=7$ ), with standard dosages $20,21,22,23,24$. After an induction of anesthesia with a gaseous mixture of isoflurane, air and oxygen, the anesthetics of the four groups were as follows during rs-fMRI: - Isoflurane (Iso): the level of the isoflurane was set to $1 \%$. - Etomidate (Eto): continuous intravenous infusion at $0.5 \mathrm{mg} / \mathrm{kg} / \mathrm{min}$. - Medetomidine (Med): bolus then continuous infusion at $0.05 \mathrm{mg} / \mathrm{kg} / \mathrm{h}$. - Urethane (Ure): bolus of $1.25 \mathrm{~g} / \mathrm{kg}$ injected intraperitoneally. To evaluate whether animal strain bias this comparison, Isoflurane was also administered to Wistar rats (Iso-W, $\mathrm{n}=7$ ). Overall, five groups were evaluated. During the experiments, a warm water circulating under the animal was regulated to maintain the body temperature to a target level of $37{ }^{\circ} \mathrm{C}$ inside the MRI device. A group of

Dead rat was also recorded for comparison (Dead, $\mathrm{n}=4$ ), one hour after a lethal dose 
of pentobarbital.

\subsection{MRI acquisitions at 9.4 $T$ and data processing with wavelets}

rs-fMRI was performed using a single shot, echo-planar, imaging with $\mathrm{TR} / \mathrm{TE}=$ $500 / 20 \mathrm{~ms}$, spatial resolution of $0.47 \times 0.47 \times 1.1 \mathrm{~mm}$, and 3600 repetitions $(30 \mathrm{~min})$. fMRI BOLD signals were first extracted on $\mathrm{p}=51$ brain areas from a home-made atlas based on published ones [25, 26] after normalization of anatomical images and co-registration of fMRIs. These operations were applied using Statistical Parametric Mapping software (SPM12) [27]. The Tohoku template was taken as the reference with bregma at zero coordinate, at the apex of the most forward crossing fibers of the anterior commissure. Classical preprocessing procedures as implemented in SPM12 [27] were applied: normalization of anatomical images; co-registration of fMRIs; signal extraction by weighting the activity of voxels in an area, for each of the 51 areas of the atlas (additional details can be found in an accompanying paper [28]). Motions were detected using the framewise displacement (FD) [29] with the parameters obtained from the coregistration of fMRI to the first image in the fMRI sequence.

BOLD signals were decomposed with wavelet transforms, with a maximal overlap discrete wavelet transform (modwt) at different scales and Daubechies orthonormal compactly supported wavelets of length $\mathrm{L}=8$, using the wavedec package [2]. In this study, only detail coefficients at scales 1 to 6 (cD1 - cD6) are retained, with frequency bands in Hz: $0.016<\mathrm{cD} 6<0.031<\mathrm{cD} 5<0.063<\mathrm{cD} 4<0.125<\mathrm{cD} 3<0.25<$ $\mathrm{cD} 2<0.5<\mathrm{cD} 1<1$, with a particular focus on the $\mathrm{cD} 4$ band. The signals were motion corrected using a scrubbing approach, i.e., removing the samples artefacted by movements while applying the temporal influence of the wavelet filtering at each scale. We applied a scrubbing method with boxcar filtering [30 taking into account an hemodynamic response function of $20 \mathrm{~s}$ and wavelet support at different scales. Only five rats showed major displacements, FD over $0.47 \mathrm{~mm}$ for more than $1 \%$ of the sample size, in two groups of rats Iso-L and Ure-L (Iso-L (3, 5, 46\%), Ure-L (6, 24\%)). Correlations were performed on these signals to evaluate FC and other graph measures.

\subsection{Detection of significant correlations using wavelets}

A model of uncorrelated random noises was used to obtain significant thresholds at a symmetrical initial $95 \%$ confidence interval. Because of the scrubbing based on FD, signals obtained on different rats have not the same size. Besides, non-successive samples are retained. But, since the retained samples already takes into account the influence of the wavelet support, the following formula [31, 32, 33] was used to obtain the limits of significant correlations:

$$
\rho_{m, M}=\tanh \left(h(\rho) \pm \frac{\Phi^{-1}(1-p)}{\sqrt{\left(\frac{N_{r}}{2^{j}}-3\right)}}\right)
$$


where $N_{r}$ is the number of samples at wavelet scale $j, h(\rho)=\tanh ^{-1}(\rho)$ and $\Phi^{-1}(p)$ is the $p$ th quantile of the standard normal distribution. To take into account the false detection rate introduced by the repetition of tests across the 51 retained brain areas present in the atlas, a Bonferroni correction was applied to the $1275=(51 \times 50 / 2)$ pairwise correlations, leading to correct the significance level to $p_{b f}=0.025 / 1275=1.96 e-5=0.002 \%$. For comparison, sets of 51 signals with 3600 samples with i.i.d. samples following a Gaussian distribution $N(0,1)$ were simulated and filtered.

\subsection{Networks measures and simulations}

The functional connectivity corresponds to the correlation of the signals obtained from wavelet coefficients at a given scale [2] between pairs of areas. It is given by:

$$
C(i, j)=\frac{\frac{1}{N} \sum_{k=1}^{N} \bar{x}_{i}(k) \bar{x}_{j}(k)}{\left(\frac{1}{N} \sum_{k=1}^{N} \bar{x}_{i}^{2}(k) \frac{1}{N} \sum_{k=1}^{N} \bar{x}_{j}^{2}(k)\right)^{1 / 2}}
$$

with $\bar{x}_{i}$ the centered variable, i.e. the variable with its sample mean subtracted, for area $i$ over $N$ temporal samples.

From this FC and the networks associated, we extracted graph measures usually used in fMRI studies [2, 34, 35, 36, 37, 38, 39, 40, 41, 42, 43, 44, 45]: degree, local and global efficiencies, clustering coefficient, betweenness and closeness centralities. These graph measures were computed to evaluate the effects of anesthesia and were compared to mathematical models like random or small-world networks.

2.4.1. Graphs measures Let $\mathcal{G}=(V, E)$ be the undirected graph associated to the binarized thresholded correlation matrix $C$, with $V$ the set of vertices or nodes and $E$ the set of edges. The number of vertices or nodes, noted p, corresponds to the number of brain areas in this study $(\mathrm{p}=51)$. By varying the thresholds value applied to the correlation matrix, different graphs $\mathcal{G}$ were obtained. All self-connections were set to 0 . Absolute values of correlations were considered here, meaning that negative values

of correlations (anticorrelations) were treated as positive values. Let neM $=\frac{\mathrm{p}(\mathrm{p}-1)}{2}$ be the maximal number of edges in the undirected graph $\mathcal{G}$ without self-connections. The density of the graph is defined as $\delta=\frac{\text { ne }}{\text { neM }}$, ne the ratio of edges present in the graph over neM the maximal number of possible edges between vertices (fully connected graph). Measures can be computed on this unweigthed undirected graph. Different measures were evaluated: degree (deg), clustering coefficient (clu), global efficiency (glo), local efficiency (loc) and betweenness centrality (bet) [44]. The degree is defined as,

$$
\operatorname{deg}_{i}=\sum_{j} A_{i j}
$$

where $A$ is the adjacency matrix of the graph with $A_{i j}=1$ if there is a connection between nodes $i$ and $j$ and 0 otherwise.

For all $i, 1 \leq i \leq \mathrm{p}$, the clustering of node $i$ is defined as,

$$
\operatorname{clu}_{i}(\mathcal{G})=\frac{2 \operatorname{tri}_{i}}{\operatorname{deg}_{i}\left(\operatorname{deg}_{i}-1\right)},
$$


where $\operatorname{tri}_{i}$ is the number of triangles including node $i$. For all $i, 1 \leq i \leq \mathrm{p}$, the global efficiency of node $i$ is defined as,

$$
\mathrm{glo}_{i}(\mathcal{G})=\frac{1}{(\mathrm{p}-1)} \sum_{j}^{\mathrm{p}} \frac{1}{l(i, j)},
$$

where $l(i, j)$ is the shortest path, if available, between node $i$ and node $j$, in terms of the $\mathrm{p}$ nodes, in the undirected graph $\mathcal{G}$.

$$
\operatorname{loc}_{i}(\mathcal{G})=\frac{1}{\mathrm{p}_{i}} \sum_{j \in \mathcal{G}_{i}} \operatorname{glo}_{j}\left(\mathcal{G}_{i}\right)
$$

where $\mathcal{G}_{i}$ is the subgraph made with the $\mathrm{p}_{i}$ neighbors of node $i$. Local efficiency of node $i$ is the average global efficiency of the subgraph of neighbors of $i$.

$$
\text { bet }_{i}=\sum_{j, k \in \mathcal{G}} \frac{\sigma(j, k \mid i)}{\sigma(j, k)},
$$

where $\sigma(j, k)$ is the number of shortest paths from $j$ to $k$, and $\sigma(j, k \mid i)$ is the number of those paths involving $i$.

Global measures quantifying topological properties of the whole networks were obtained by taking the average of these local measures over the set of nodes of $\mathcal{G}$,

$$
\text { measure }=\frac{1}{\mathrm{p}} \sum_{i} \text { measure }_{i} .
$$

Data processing and networks measures were performed in Python with package networkx [46] and personal routines using scipy [47].

2.4.2. Construction of random networks Three generative models of networks were considered for comparison with experimental networks: Barabasi-Albert (BA) [48], Erdös-Rényi (ER) [49 and Watts-Strogatz (WS) [7]. Briefly, the BA model was constructed by setting a target number of edges for each node. Each node was treated one after the other with preferential attachment to the edges already mostly connected. At the end of the process, some nodes were more connected than others with a distribution of degrees according to a power-law; the Erdös-Rényi models were constructed by giving each connection from one node to another a probability $p$; the WS model was constructed by connecting the $\mathrm{k}$ nearest neighbors of each node in a ring graph of $\mathrm{n}$ nodes, then by connecting with a probability parameter $p$ (WS $p$ ) the $\mathrm{k}$ nearest neighbors to other nodes in the graph.

Simulations were performed in Python with package igraph [50]. For the BA model, the default psumtree option was used to generate the simulated networks. The number of outgoing edges varied from 1 to 51. For the ER model, the parameter for the probability of edges varied from 0 to 1 with a step of 0.01 . For the WS model, the dimension was set to 1 and the size to 51. The rewiring probability was taken in the set $[0,0.01,0.05,0.1,0.5,0.9]$ and the distance within which two vertices are connected varied from 0 to 51 , thus increasing the density of the network. 


\subsection{Data availability}

Data are available at DOI. 10.5281/zenodo.2452871.

\section{Results}

\subsection{Distribution of correlation coefficients: simulated noises versus anesthetized or dead rats}

To evaluate the functional connectivity in the brain, correlations of fMRI signals from different areas is a common practice and is used in several publications [51], but few studies evaluate whether correlation values obtained from different areas are significant or due to spurious correlations. Two issues need to be evaluated to determine the statistical significance of the values in such situation:

- influence of the number of samples for the estimation of the correlation between two signals; Applying filtering to signals, using wavelet decomposition for example in this study, changes the size of uncorrelated samples.

- influence of test repetition due to multiple pairwise comparisons of signal between areas;

For the first point, examples of simulated distributions of correlation values for $\mathrm{p}$ i.i.d uncorrelated Gaussian processes (GP) from $N$ samples are given in Fig. 11a) and b) in terms of probability density functions (PDF) and cumulative distribution functions $(\mathrm{CDF})$. Histograms are estimated on 100 bins in the interval [-1, 1], with mean (M) and standard error of the mean (SEM) evaluated over 1000 trials. The number $N$ controls the shape of the distributions while the number $\mathrm{p}$ influences the variance of the estimates. The larger the number of samples is, the more pronounced the PDF peaks around 0 and the closer the CDF is to a hard sigmoid function around 0 , as shown in Fig. 17. The theoretical parametric probability density functions for these correlation coefficients computed on multidimensional random variables are not simple to obtain and can be found in [33], see in appendix Eq.A.1. A test of significance of correlation values, rejecting the null hypothesis that the correlation is obtained from an i.i.d Gaussian multivariate, can then be proposed on the basis of this distribution as detailed in 2.3. The second point relates to multiple comparisons and can be resolved by using multiple testing procedures such as the classical Bonferroni [16]. The PDF of correlation values between brain areas obtained on the different groups of anesthetized rats at different coefficient details and at level $i\left(c D_{i}\right)$ are shown in Fig. 1 $\mathrm{d}$. PDF of correlation values obtained from simulations of uncorrelated Gaussian processes (GP) are also given for comparison. Four trials are realized as in the Dead group. As the wavelet filtering reduces the number of available samples to compute the estimate by a factor of two at each scale, the number of available samples at level $c D_{i}$ is, as a first approximation $N_{i}=N / 2^{i}$. The superposition of the curves for uncorrelated GP and Dead group is almost perfect, indicating that the correlation values obtained for 

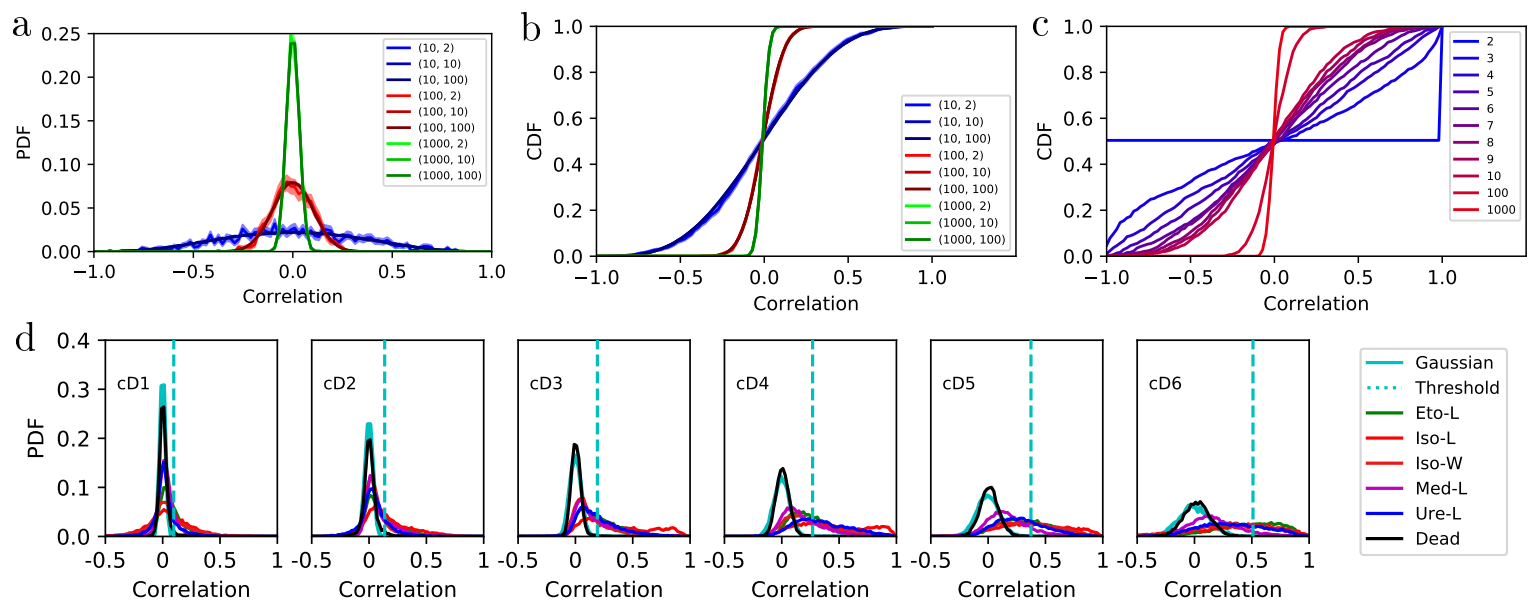

Figure 1. Distributions of correlation values for random signals and anesthetized or dead rats. a) Empirical probability density functions (PDF) of correlation values for simulated Gaussian noise while varying the number of samples and the number of signals $(N, \mathrm{p})$ given in legend. 1000 trials were used for each curve over 100 bins between $[-1,1]$, with mean \pm standard error of the mean $(\mathrm{M} \pm \mathrm{SEM})$. b) Empirical cumulative distribution functions $(\mathrm{CDF})$ of correlation values with same parameters as in a). c) Empirical CDF of correlation values for two random uncorrelated Gaussian variables while varying the number of samples for the estimates. The number of samples $N$ is shown in the legend. d) Comparison of PDF of correlations for anesthetized rats, dead rats and independent Gaussian variables with significant thresholds shown in dashed lines. Each subplot corresponds to a given wavelet frequency band: $0.016<\mathrm{cD} 6<0.031<\mathrm{cD} 5<0.063<\mathrm{cD} 4<0.125<\mathrm{cD} 3<$ $0.25<\mathrm{cD} 2<0.5<\mathrm{cD} 1<1$.

dead rats are not significantly different from those obtained for uncorrelated GP. The application of Bonferroni corrections leads to the threshold values represented by vertical blue dashed lines in Fig. 1. Few significant correlations can be found on dead rats at the different wavelet coefficient details. No more than 4 significant correlations are found for $c D_{4}$ in the group of dead rats.

For the groups of anesthetized rats, the differences between the PDFs and the initial bell shapes of the Dead and GP groups increase with the wavelet coefficient details showing that significant correlations are observed for low frequency bands [2]. The effect of the scrubbing method enables to homogenize the different groups by rejecting movement-related correlations (as shown in Fig.B1). The average correlation increases and an important number of values are over the test threshold, indicating significant differences between the values observed in anesthetized rats and the uncorrelated random variables. This confirms that the graphs extracted from live animals are significant. 


\subsection{Comparison of graphs for experimental and simulated networks}

An important number of undirected graphs can be constructed depending on the choice of correlation threshold: if we set an edge between area $i$ and $j$ when the value of correlation is higher than threshold $\theta$, i.e., $c_{i j} \geq \theta$, we obtain a graph with ne $(\theta)$ edges. With this notation, it is straightforward to obtain ne $(\theta)=(1-C D F(\theta))$ neM or $\delta(\theta)=1-C D F(\theta)$. In practice, sample CDFs are computed from sample correlation matrices, and, setting a density value $\delta$ for a graph determines the threshold value for the $100 \delta \%$ most correlated signals [2]. Fig. 2 shows the graphs obtained by thresholding the average matrices of the different groups for a density $\delta=0.1$ and wavelet scale $c D 4$ without taking into account the statistical corrections for the significance of the correlations. Distributions of degrees of the obtained graphs are also shown on Fig. 2. The 51 nodes are represented by circle sorted by degrees from 0 rad for the least connected vertex to $2 \pi \frac{50}{51} \mathrm{rad}$ for the most connected one. One color is used for edges by group of degree values. We chose to divide the possible degrees into five groups defined by their degree range: $[1],[2 ; 3],[4 ; 7],[8 ; 12]$ and $[12 ; 50]$. Edges are superimposed with the most connected edges in front of the figure. These simple representations show visually that graphs obtained under anesthesia are more structured than those obtained with Dead rats or GP simulations. Graphs and distribution of degrees for dead rats and GP are very similar, with one node for the Dead group outside the average. Note that the graph of rats are constructed with all correlation values, even those that are not significant. Working with significant values has little impact on the graphs of anesthetized rats (with a density of 0.1, all correlation values of anesthetized rats are significantly different from zero), but leads to an almost empty graph for the dead group (see Fig. 4 and appendix Fig C1).

For comparison, random networks are simulated. The selected models are ErdösRényi (ER), Barabasi-Albert (BA) and Watts-Strogatz (WS) with 51 nodes. The variations of the parameters of these models lead to graphs with comparable densities (see details in materials 2.4.2). The variation of parameter $p$ for the degree distribution in the WS model allows the exploration of small-world networks starting with lattice networks $(p=0)$ and ending with random networks $(p=1)$. Fig. 2 shows different examples of graphs at a density of approximately 0.1 , due to discrete values of the density levels obtained from simulations. The graphs and degree distributions show that all vertices are connected for simulated models ER, BA and WS, but also for the Dead group and random GP. For anesthetized rats, at density $\delta=0.1$, a fraction of vertices is not connected to the others. This clearly shows a hierarchy in the rank of correlation values, where some brain areas have higher correlations than others. Random GP simulations lead to graphs and degree distribution comparable to those of ER. Dead rats have a graph and distribution of degrees comparable to that of GP and ER. The graph of the WS model with a parameter $p>0.25$ tends towards those obtained with random GP, ER, and dead rats. The variation of the WS parameter shows differences in the construction of graphs for this model, starting with a regular network and ending 


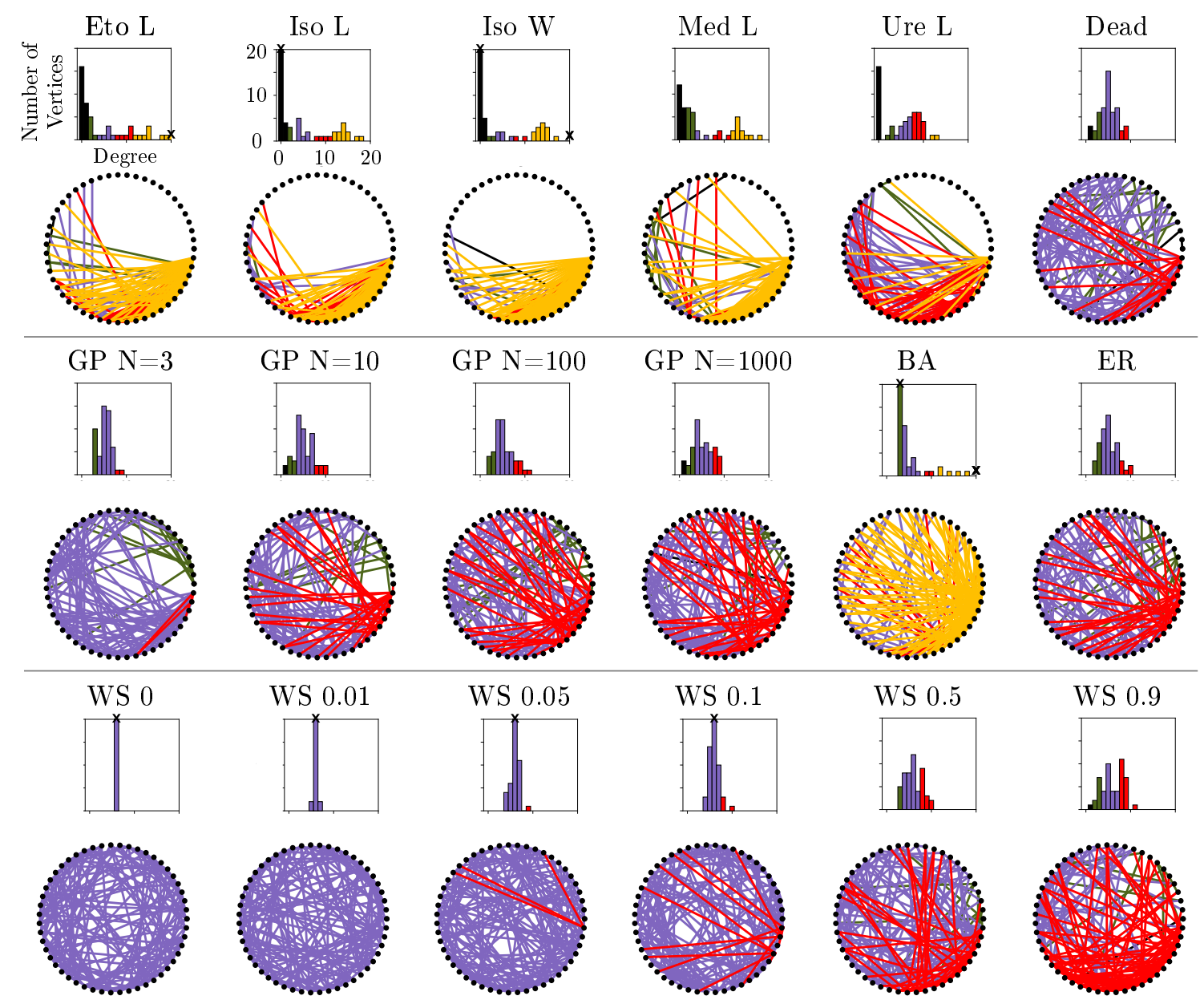

Figure 2. Graph at a density of approximately 0.1 with a circular representation and degree histograms. Each vertex of the circle is a brain area. Vertices are positioned on a circle in function of their degrees at $2 \pi \frac{i}{n_{v}}$ with $i$ the rank of its degree starting at 0 and $\mathrm{p}=51$ number of vertices. Vertex with the lowest degree is at 0 , vertex with the highest degree is at $2 \pi \frac{50}{51}$. Colors corresponds to group of degrees ([1] black, [2-3] green, [4 - 7] purple, [8 - 12] red, [12 - 50] yellow). Above circular graphs, distributions of degrees are represented with histograms. Values over 20 are represented by a $\times$.

with a random one, with an influence on the width of the degree distribution around the mode at 3 associated with the target density at $0.12=\frac{3 \times 51}{n e M}$. The BA model presents a degree distribution similar to a power law as expected, with some highly connected nodes highlighted in yellow. Graphs of anesthetized groups, with abstraction of non connected edges, are more similar to the BA graph, with more weakly connected nodes and few strongly connected ones. Iso and Med L shows a mode with some strongly connected nodes. It is difficult, from these results, to determine which generative model is the closest to the brain network graphs observed in anesthetized animals. Computation of graph measures may provide clues to characterize rat brain networks. 

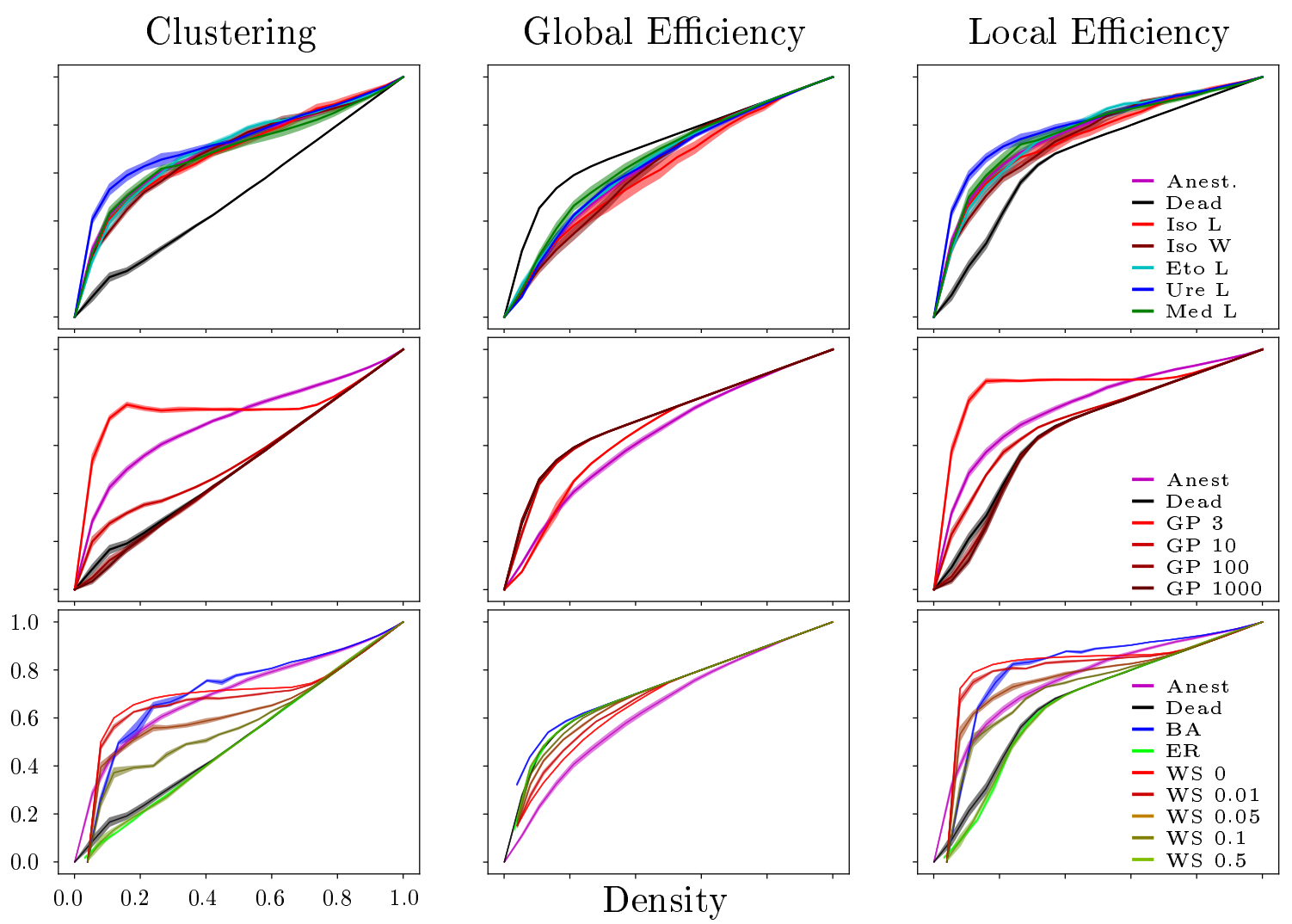

Figure 3. Graph measures in function of graph density. a-c) Groups of rats. d-f) Rats versus Gaussian processes graph measures: anesthetized rats are grouped together (Anest.), the random Gaussian processes with different $N$ used to compute correlation matrices (rand. $N$ ) g-i) Rats versus random networks measures with notations: ErdösRenyi (ER), Watt-Strogatz (WS) with a parameter of heterogeneity varying from 0 to 0.9, Barabasi-Albert (BA). a, d) clust.: clustering coefficient. b, e) eff. loc.: local efficiency. c, f) eff. glob.: global efficiency. The graph measures on the Dead group are similar to the ER or random networks. Anest. local measures are similar to BA networks (scale-free) or WS 0.1 (lightly small world) and global ones to a regular network WS 0 or random GP with small number of samples.

\subsection{Comparison of graph measures}

Graph measures can be computed for these graphs at different densities. The average values of clustering coefficient, local and global efficiency are computed to assess for the small-worldness, heterogeneity or randomness of networks [44]. The computation of these values is presented in 2.4. Fig. 3 shows different graph measures obtained from anesthetized or Dead rats, GP and structural random networks. Fig. 3 a-c indicates that the different groups of anesthetized rats show similar global measures as a function of the density. There is a clear difference between dead rats and anesthetized rats. The group of Dead rats has low clustering values, high global efficiency, and low local efficiency at low densities. The Ure L group shows a higher clustering and local efficiency at low densities than the other groups. For the other figures, anesthetized rats (Anest.) 
corresponds to the average values of these groups.

Fig. 3d-f compare graph measures for simulated uncorrelated GP using a range of numbers of samples with dead and anesthetized rats. Spurious results may be obtained when the number of samples is too small. In Fig. 3e, the graph obtained with 3 samples out of 51 GP may generate a local efficiency similar to the one obtained with Anest. but with a global efficiency that differs from the one reported in Fig. 33. With $N=100$ and higher, the different curves obtained are largely superimposed on the curves of the Dead group, showing once again that the graph of dead rats corresponds to that obtained from uncorrelated Gaussian noise.

For theoretical models, the parameters of the simulated examples are set so as to be comparable to the graphs constructed using experimental data i.e. by taking 51 nodes. The number of simulated examples is set to 4 after observing that the dispersion of simulations is low. Measures are also evaluated for densities of the simulated graphs associated to 20 bins in the interval $[0,1]$. Fig. 3 3 -i display comparisons with measures obtained using various generative models of networks. Dead group curves are largely superimposed on curves from ER, with a difference for low densities. The variations of the WS models converge to the curves obtained for ER and Dead groups. Anest. group measures for clustering and local (global) efficiency have comparable behaviors to the ones observed for BA, regular networks WS 0 and WS $\leq 0.1$ (regular and small world) with a match on local efficiency with WS 0.1. However, global efficiency measures evaluated on the Anest. group are different from the generative model. At high densities, all graph measures converges to the same values, driven by the fully connected network.

\subsection{Effect of anesthetics on the spatial organization of the network}

In order to assess the local impact of anesthetics on nodes, different measures of nodes are computed and spatially represented according to the anatomical location of the area centers of gravity $(\mathrm{COG})$. The $\mathrm{COG}$ are projected into three different directions on the left-right (LR) axis, posterior-anterior (PA) axis, and inferior-superior (IS) axis. A representation of these projections are given in Fig. D4 with a list of abbreviations and area names. Fig. 4 presents a selection of measures on LR-IS projections. Other projections are proposed in Appendix D. Computations of nodes measures are given in 2.4. Values are computed for each rat in each group with only significant correlation values for the graph obtained at a density of 0.1 , and then averaged. The degree measure reveals higher connection values in the superior and anterior areas than in the inferior and posterior ones (see PA projections given in appendix Fig. D2 and D3.). This indicates that, at this density, some areas are more connected than others depending on the anesthesia. For example, the degree of ACC is lower for Ure-L and Iso-L than for other anesthetics. In Ure-L, the degree distribution is more uniform and exhibits values in subcortical areas comparable to those in cortical areas. Global efficiency seems uniform in all areas for all anesthetics, but some subtleties can be observed for some areas, especially for PA projections, see Fig. D2 and D3, Local efficiency leads to 

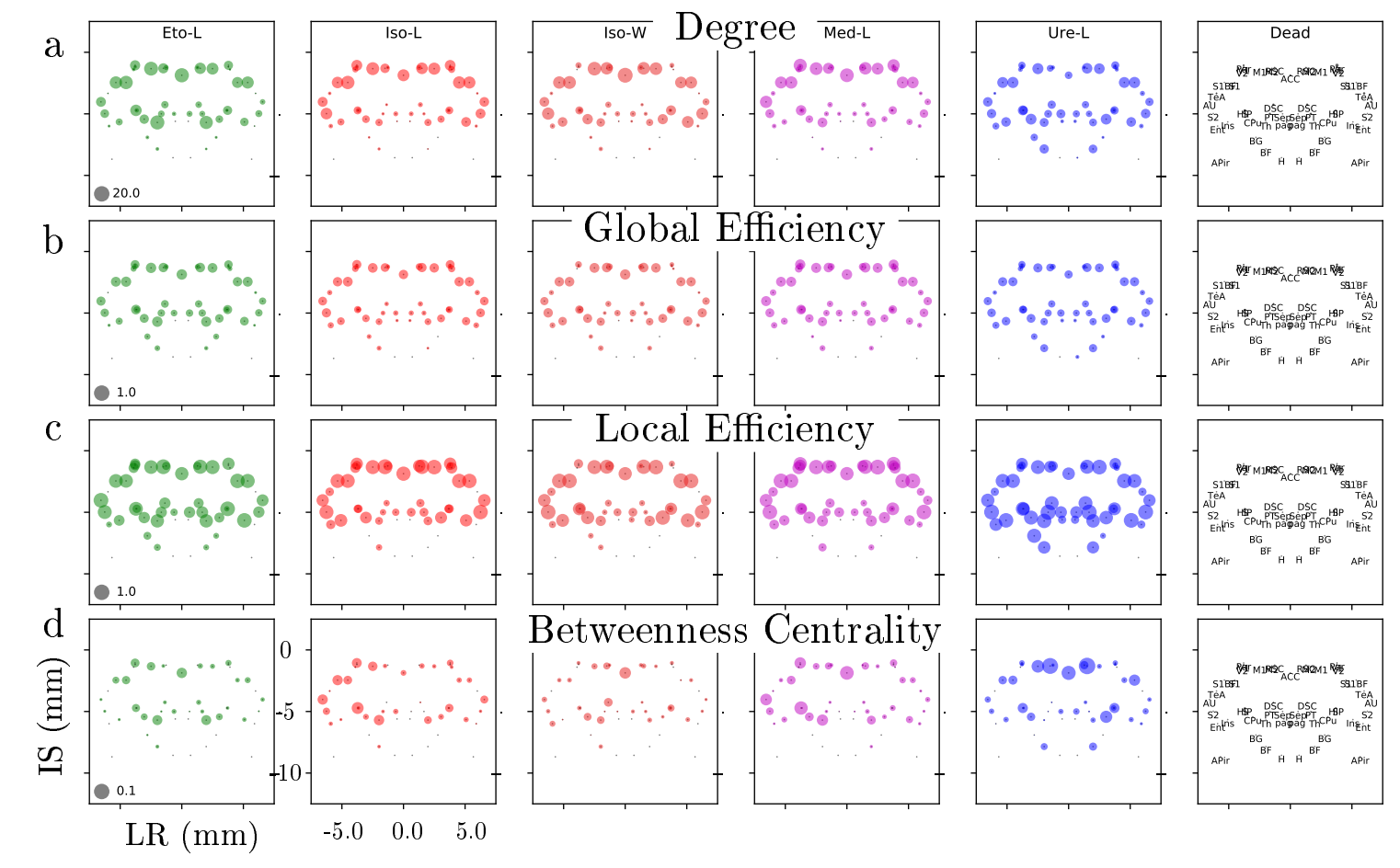

Figure 4. Graph measures per nodes in LR-IS. All measures are computed on significant edges from graphs with a density of 0.1. a) Degree. b) Global efficiency. c) Local efficiency. d) Betweenness centrality. A list of areas is available in appendix Fig. D4.

comparable measures and suggests that subcortical areas are less clustered or locally less efficient than cortical areas. Only the Ure-L group shows a preserved local efficiency in both cortical and subcortical areas (a uniform clustering as shown in Fig. D1.D3). For betweenness centrality, some important nodes are influenced by anesthetics, such as ACC. The comparison of areas measures reveals the influence of anesthetics, for example: ACC betweenness centrality is medium or strong in Med-L, Iso-W and Eto-L, with a high degree, potentially indicating that this area is not really altered by this anesthetic; ACC betweenness centrality is strong in Ure-L even with a small degree, indicating the central role of this area in the network and the alteration induced by this anesthetic; ACC betweenness is weak in Iso-L with a medium degree indicating its moderate role in the network and the influence of the anesthetic on it. These results show the complementarity of the graph measures to characterize the role of each area of the brain. 


\section{Discussion}

\subsection{Importance of significant correlation coefficients}

Construction of brain networks from functional connectivity matrices has raised the difficult issue of threshold choice [11. Indeed, it is still unclear whether there is a better choice between weighted or unweighted graphs. From a statistical point of view, it is evident that depending on the size of the data, strong spurious correlations can be observed [16]. It is obviously illustrated in Fig. 1 1a-c by simulated distributions of correlation coefficients from uncorrelated Gaussian processes, where the number of samples plays an important role [8]. We advocate in this article that these spurious correlations be set to zero and not be taken into account in the construction of the networks as in Fig. 4. Indeed, it is important to consider the effect of data filtering, commonly used in fMRI. As shown in Fig. 1 $1 \mathrm{~d}$ of our study, wavelet filtering reduces the number of samples used to evaluate correlation and thus may create spurious correlations. This is also valid for any other filtering strategy and must be taken into account. This enables the test parameter to be defined to decide whether a correlation value is different from those obtained theoretically from uncorrelated random multivariates with or without simplification of the initial parametric model [33 as shown in Appendix A. An other implication of filtering is to remember that a simple instantaneous movement on one sample can propagate throughout the duration of the impulse response of the filter. The rejection of these spurious samples leads to a reduction in the number of available samples and thus, as shown in Fig. 1, an increase in the detection threshold for significant correlations. The use of a scrubbing method and wavelet filtering allows the precise exclusion of corrupted data and the avoidance of spurious correlations(further analyses of the impact of head motions are developed in [30]). We observed that the influence of the depth of anesthesia, regulated by the dose, was an important factor to control and avoid motion artefacts. It was observed in all groups and especially in the Iso-L and Ure-L groups.

In addition to the sample size, the statistics provide a framework for taking into account multiple comparisons. One solution is to control for family-wise error rate with the Bonferroni procedure, as proposed here. This procedure is highly restrictive and future work is necessary to improve the detection of edges of the graph. Discoveries in the field of high dimensional correlation detection as discussed in [16 may be an interesting alternative.

The influence of false detection of graph edges is a very important topic highlighted in recent studies [18, 17]. The problem relies not only in the edge detection, but also in biased estimation of the weights. This is particularly well illustrated in a simulation example [18] where classical graph measures such as betweenness or closeness centrality are computed on a given artificial graph where all nodes are equivalent. It is shown that, using simulations of Gaussian processes, these graph measures can discriminate the nodes of the networks and wrongly classify them as hubs. It is shown in Fig. C1 that from the Dead group, GP, WS with a high parameter of reconnection $p$, or ER 
networks, the degree distribution leads to nodes with high values. In many fMRI studies, the objective is to evaluate the connectivity between different brain regions in order to characterize their role in a targeted disease. This is the purpose of this study where anesthetics are delivered to rats to observe their influence on the brain connectivity and is illustrated in Fig. 4 where differences between graph measures are observed in some areas. Unweighted graphs are considered here in order to limit the influence of bias on weights. Another motivation for the use of anesthetics on rats is the possibility to have long acquisitions and use known hypotheses on the role of anesthetics. For a review of the different already proposed methods used in rodents under anesthesia, we refer the reader to $[52,36,53,54,55,56,28]$ where alterations of cortico-cortical, subcorticocortical, intra and inter hemispheric connections are modified, with a dependence on the anesthesia regime at different levels. There are several methodologies used in these different papers that make comparisons difficult. This is also the case in rs-fMRI studies in human, where average measures are proposed [38, 8, 57, 45, 58, 59] with some giving variations in function of densities, and here also a plethora of definitions. One of the main difference with our rat brain connectivity study is the size of the samples. In our study the acquisitions are generally longer than the other cited references, from three to six times longer (except [55]). This allows us to investigate the robustness of the brain connectivity extractions and the possibility to compare different anesthetics. The characterisation of rat brain connectivity at the regional level is still under investigations with no clear consensus. Along with our study, to the best of our knowledge, two other papers extracted regional graph measures [60, 36] using different definitions of measures, different areas, different dose of MED. Hubs were identified in these two papers: in 36 ACC, Hip, CPu, M2, S1, RSC, Ins using MED, and in [60 Hip, CPu, Th, left more than right using ISO. In our study, the hubs identified in MED group are ACC, Th, Par, Hip, AU and in ISO group Hip, $\mathrm{CPu}$, Th, AU, Par, S1BF, left more than right. Common regions are identified in our study and already published work. However, the comparison with already published works is hard to evaluate and out of the scope of this engineering study, though seems coherent with major observations on principal brain areas and is coherent with what is expected with random networks. Further investigations are needed to compare brain graph connectivity under anesthetics.

\subsection{Comparison of rat brain networks with generative models}

The comparison of networks of anesthetized rats to simulations of generative models is providing a different approach to highlight specific graph topologies. To our knowledge this is the first study to compare different generative models to brain real data on a large range of density and using different graphs measures. Interestingly, Fig. 3 shows that taking a global measure on the network, the anesthetized rats present homogeneous values for all the computed measures and on the whole range of densities. It is not possible in our experiments to find a single generative model to match the observed brain networks of anesthetized rats for all the measures. Alternative of generative 
models should be considered [4]. The behaviour of GP with a very small number of samples is not clearly understood and the closeness of the GP 3 graphs with brain networks should be explored further. Fig. 3 shows also that the curves of the group of Dead rats are consistently close to those of GP 1000 for all measures and the whole range of density but slightly different from simulated random networks [8]. It may be more appropriate to compare obtained rs-fMRI brain networks to simulated networks constructed using uncorrelated Gaussian noise rather than ER [44].

Fig. 2 shows that the comparison of generative models with Anest. group is biased for low values of densities. Indeed, all simulated generative models have no disconnected nodes, i.e. all nodes have at least one edge. This is not the case for the experimental groups where networks at low densities presents nodes without connections. This has already been observed on human data sets [61]. Graph measures are known to be sensitive to this specific features [62] and preprocessing may have to be applied to correct for this. In order to improve the comparison, other models with varying the number of connected edges must be developed and tested. The comparison and some behaviors in measures could be explained by these differences.

\subsection{Evaluation of regional measures}

The analysis of graph has brought a huge number of measures to asses for nodes influence [44]. Usually, the choice of graph measures is dependent on the study and highlight specific features of the nodes of the graphs [6]. In this study, we have focused on the local and global efficiencies, as in [2], and it is shown in Fig. D1p, f that the information brought by clustering and closeness centrality does not bring more information than those presented in Fig. 3e,f [44]. The betweenness centrality, computed on unweighted graph seems to be an important complementary measure to assess for the influence of a node on the graph and especially to emphasize hubs [9]. Concerning Anesth. rats, it is important to note that a high connectivity is still present in all networks, as observed in Fig. 3d, with modifications of hubs due to the local effects of anesthetics. An example of the action of Ure on ACC, leading to a diminution in the global efficiency of this hub, is presented. The influence of anesthesia on the IS and AP axis is displayed on Fig. D1.D3. From this observation, we can not conclude if this is due to an artefactual measure noise due to the influence of the position of the sensor in the MRI located in the upper part, or a real effect due to the action of anesthesia on subcortical and parietal areas. In addition, a high value of degree for Ure L and Iso L group can be potentially related to the level/type of anesthesia (also observed in Fig 1). Indeed, the level of anesthesia, or consciousness, should be taken in consideration when studying brain connectivity networks as already mentioned in $63,64,53,55,56,36,52$.

In this study, we focus on exploring graph measures for unweighted graphs at a given density. The use of weighted measures is hard to evaluate with correlation values in $[0,1]$, and leads to distances difficult to interpret, possible miscalculation of short paths, or other graph measures, as discussed in [44]. It would be of interest to develop 
methods to work directly on the correlation matrices, eliminating non significant values. Indeed, nonsignificant values of correlations can be used to obtain reference measures associated to random networks as with smallworldness proposed in [65, 44], but not evaluated in this study. Spurious values obtained from Dead rats can be compared with those from anesthetized rats as proposed in Fig.D1,D3, but need more development to yield significant conclusions.

\section{Conclusion}

Construction of brain graphs based on functional connectivity is valid when applying rigorous statistical methods. Wavelets and control of multiple comparisons provide efficient tools to extract significant brain graphs. We validate these methods on simulations and real data from dead rats where brain graphs from dead rats are found equivalent to those obtained with uncorrelated Gaussian noise. Extensive comparisons with generative models confirm the differences between simulated models and anesthetized rats. Finally, graph measures allow to capture topological roles of brain areas associated with the use of anesthetics in rats. FMRI signals are very complex and future work is needed to better understand the changes in correlations associated with anesthetics. In particular, these differences may be related to altered hemodynamic response and adequate statistical estimation should be proposed to take into account the spatiotemporal nature of the data sets.

\section{Acknowledgements}

The authors wish to thank Florent Chatelain for invaluable discussions and orientations concerning multivariate statistical methods. The MRI facility IRMaGe is partly funded by the French program Investissement d'Avenir run by the French National Research Agency, grant Infrastructure d'avenir en Biologie Santé [ANR-11-INBS-0006]. This project was partly funded by NeuroCoG IDEX UGA in the framework of the Investissements d'avenir program [ANR-15-IDEX-02].

\section{References}

\section{References}

[1] Olaf Sporns. Discovering the human connectome. MIT press, 2012.

[2] Sophie Achard et al. "A Resilient, Low-Frequency, Small-World Human Brain Functional Network with Highly Connected Association Cortical Hubs". en. In: The Journal of Neuroscience 26.1 (Jan. 2006). 00831, pp. 63-72. ISSN: 0270-6474, 1529-2401. URL: https://doi.org/10.1523/JNEUROSCI .3874-05.2006. 
[3] Fabrizio De Vico Fallani et al. "Graph analysis of functional brain networks: practical issues in translational neuroscience". In: Philosophical Transactions of the Royal Society B: Biological Sciences 369.1653 (2014), p. 20130521. URL: https://doi.org/10.1098/rstb.2013.0521.

[4] Petra E Vértes et al. "Simple models of human brain functional networks". In: Proceedings of the National Academy of Sciences 109.15 (2012), pp. 5868-5873. URL: https://doi.org/10.1073/pnas.1111738109.

[5] Jonas Richiardi et al. "Machine learning with brain graphs: Predictive modeling approaches for functional imaging in systems neuroscience". In: IEEE Signal Processing Magazine 30.3 (2013), pp. 58-70. URL: https://doi.org/10.1109/ MSP. 2012.2233865.

[6] E. Bullmore and O. Sporns. "Complex brain networks: graph theoretical analysis of structural and functional systems." In: Nat Rev Neurosci 10.3 (Mar. 2009), pp. 186-198. DOI: http://dx.doi.org/10.1038/nrn2575.

[7] Duncan J Watts and Steven H Strogatz. "Collective dynamics of 'small-world' networks". In: nature 393.6684 (1998), p. 440. URL: https://doi.org/10.1038/ 30918.

[8] Andrew Zalesky, Alex Fornito, and Ed Bullmore. "On the use of correlation as a measure of network connectivity". In: NeuroImage 60.4 (2012), pp. 2096-2106. URL: https://doi.org/10.1016/j.neuroimage.2012.02.001.

[9] Mark Newman. Networks. Oxford university press, 2018.

[10] Nikola T Markov et al. "Cortical high-density counterstream architectures". In: Science 342.6158 (2013), p. 1238406. URL: https://doi.org/10.1126/science. 1238406 .

[11] Danielle S Bassett and Edward T Bullmore. "Small-world brain networks revisited". In: The Neuroscientist 23.5 (2017), pp. 499-516. URL: https://doi . org/10.1177/1073858416667720.

[12] Ameera X Patel and Edward T Bullmore. "A wavelet-based estimator of the degrees of freedom in denoised fMRI time series for probabilistic testing of functional connectivity and brain graphs". In: NeuroImage (2015). URL: https: //doi.org/10.1016/j.neuroimage.2015.04.052.

[13] Karina Ashurbekova, Sophie Achard, and Florence Forbes. "Structure Learning via Hadamard Product of Correlation and Partial Correlation Matrices". In: EUSIPCO 2019 - 27th European Signal Processing Conference, Sep 2019, A Coruña, Spain. 2019. URL: https : // doi . org/10 . 23919 / EUSIPCO . 2019 . 8902948.

[14] František Váša, Edward T Bullmore, and Ameera X Patel. "Probabilistic thresholding of functional connectomes: Application to schizophrenia". In: Neuroimage 172 (2018), pp. 326-340. URL: https : //doi .org/10 .1016/j . neuroimage.2017.12.043. 
[15] Maïté Termenon et al. "Reliability of graph analysis of resting state fMRI using test-retest dataset from the Human Connectome Project". In: Neuroimage 142 (2016), pp. 172-187. URL: https://doi.org/10.1016/j.neuroimage.2016.05. 062.

[16] Alfred Hero and Bala Rajaratnam. "Large-scale correlation screening". In: Journal of the American Statistical Association 106.496 (2011), pp. 1540-1552. URL: https://doi.org/10.1198/jasa.2011.tm11015.

[17] Qikai Niu et al. "Robustness of centrality measures against network manipulation". In: Physica A: Statistical Mechanics and its Applications 438 (2015), pp. 124-131. URL: https://doi.org/10.1016/j.physa.2015.06.031.

[18] Sacha Epskamp, Denny Borsboom, and Eiko I Fried. "Estimating psychological networks and their accuracy: A tutorial paper". In: Behavior Research Methods 50.1 (2018), pp. 195-212. URL: https://doi.org/10.3758/s13428-017-0862-1.

[19] Han Lv et al. "Resting-state functional MRI: everything that nonexperts have always wanted to know". In: American Journal of Neuroradiology 39.8 (2018), pp. 1390-1399. URL: https://doi.org/10.3174/ajnr.A5527.

[20] Karl J Field, William J White, and C Max Lang. "Anaesthetic effects of chloral hydrate, pentobarbitone and urethane in adult male rats". In: Laboratory animals 27.3 (1993), pp. 258-269. URL: https : / / doi . org / 10 . 1258 \% 2F002367793780745471.

[21] Luisa Ciobanu et al. "Effects of anesthetic agents on brain blood oxygenation level revealed with ultra-high field MRI". In: PloS One 7.3 (2012), e32645. URL: https://doi.org/10.1371/journal.pone.0032645.

[22] Kun Wang et al. "Temporal scaling properties and spatial synchronization of spontaneous blood oxygenation level-dependent (BOLD) signal fluctuations in rat sensorimotor network at different levels of isoflurane anesthesia". In: NMR Biomed. 24.1 (2011), pp. 61-67. ISSN: 1099-1492. URL: https://doi.org/10. 1002/nbm.1556.

[23] Jennifer X Haensel, Aisling Spain, and Chris Martin. "A systematic review of physiological methods in rodent pharmacological MRI studies". In: Psychopharmacology 232.3 (2015), pp. 489-499. URL: https://doi.org/10.1007\%2Fs00213014-3855-0.

[24] Marija M Petrinovic et al. "A novel anesthesia regime enables neurofunctional studies and imaging genetics across mouse strains". In: Sci. Rep. 6 (2016), pp. 2045-2322. URL: https://doi.org/10.1038/srep24523.

[25] Pedro Antonio Valdes Hernandez et al. "An in vivo MRI template set for morphometry, tissue segmentation, and fMRI localization in rats". In: Front. Neuroinf. 5 (2011), p. 26. URL: https://doi.org/10.3389/fninf.2011.00026. 
[26] Eszter A. Papp et al. "Waxholm Space atlas of the Sprague Dawley rat brain". In: NeuroImage 97 (2014), pp. 374-386. ISSN: 1053-8119. URL: https ://doi.org/ $10.1016 / \mathrm{j}$.neuroimage.2014.04.001

[27] John Ashburner et al. "SPM12 manual". In: Wellcome Trust Centre for Neuroimaging, London, UK (2014). URL: https : / / www . fil . ion . ucl . ac . uk/spm/doc/spm12_manual.pdf.

[28] Guillaume J-P C Becq et al. "Functional connectivity is preserved but reorganized across several anesthetic regimes". In: Neurogimage (2020). URL: https://doi. org/10.1016/j.neuroimage.2020.116945.

[29] Jonathan D Power et al. "Spurious but systematic correlations in functional connectivity MRI networks arise from subject motion". In: Neuroimage 59.3 (2012), pp. 2142-2154. URL: https://doi.org/10.1016/j.neuroimage. 2011. 10.018 .

[30] Chao-Gan Yan et al. "A comprehensive assessment of regional variation in the impact of head micromovements on functional connectomics". In: Neuroimage 76 (2013), pp. 183-201. URL: https://doi.org/10.1016/j.neuroimage.2013.03. 004.

[31] Brandon J Whitcher. Assessing nonstationary time series using wavelets. PhD Thesis, University of Washington, 1998. URL: http://citeseerx.ist.psu.edu/ viewdoc/download?doi=10.1.1.29.5644\&rep=rep1\&type=pdf.

[32] Brandon Whitcher, Peter Guttorp, and Donald B Percival. "Wavelet analysis of covariance with application to atmospheric time series". In: Journal of Geophysical Research: Atmospheres 105.D11 (2000), pp. 14941-14962. URL: https : //doi . org/10.1029/2000JD900110.

[33] Robb J Muirhead. Aspects of multivariate statistical theory. Vol. 197. John Wiley \& Sons, 2009.

[34] Sophie Achard and Ed Bullmore. "Efficiency and cost of economical brain functional networks". In: PLoS computational biology 3.2 (2007), e17. URL: https: //doi.org/10.1371/journal.pcbi.0030017.

[35] Michael W. Cole, Sudhir Pathak, and Walter Schneider. "Identifying the brain's most globally connected regions". In: NeuroImage 49.4 (2010), pp. 3132-3148. ISSN: 1053-8119. URL: https://doi.org/10.1016/j .neuroimage.2009.11.001.

[36] Dany V D'Souza et al. "Preserved modular network organization in the sedated rat brain". In: PLoS One 9.9 (2014), e106156. uRL: https://doi.org/10.1371/ journal.pone.0106156.

[37] R. Matthew Hutchison et al. "Isoflurane induces dose-dependent alterations in the cortical connectivity profiles and dynamic properties of the brain's functional architecture". In: Human Brain Mapping 35.12 (2014), pp. 5754-5775. ISSN: 10970193. URL: https://doi.org/10.1002/hbm.22583. 
[38] Xia Liang et al. "Effects of different correlation metrics and preprocessing factors on small-world brain functional networks: a resting-state functional MRI study". In: PloS one 7.3 (2012), e32766. URL: https : //doi .org/10.1371/journal. pone.0032766.

[39] Adam Liska et al. "Functional connectivity hubs of the mouse brain". In: Neuroimage 115 (2015), pp. 281-291. URL: https://doi.org/10.1016/j . neuroimage.2015.04.033.

[40] Hanbing Lu et al. "Rat brains also have a default mode network". In: Proceedings of the National Academy of Sciences 109.10 (2012), pp. 3979-3984. URL: https: //doi.org/10.1073/pnas.1200506109.

[41] Zhiwei Ma et al. "Functional atlas of the awake rat brain: A neuroimaging study of rat brain specialization and integration". In: Neuroimage (2016). URL: https: //doi.org/10.1016/j.neuroimage.2016.07.007.

[42] Matthew Magnuson, Waqas Majeed, and Shella D Keilholz. "Functional connectivity in blood oxygenation level-dependent and cerebral blood volumeweighted resting state functional magnetic resonance imaging in the rat brain". In: Journal of Magnetic Resonance Imaging 32.3 (2010), pp. 584-592. URL: https: //doi.org/10.1002/jmri.22295.

[43] Matthew Evan Magnuson et al. "Time-dependent effects of isoflurane and dexmedetomidine on functional connectivity, spectral characteristics, and spatial distribution of spontaneous BOLD fluctuations". In: NMR in biomedicine 27.3 (2014), pp. 291-303. URL: https://doi.org/10.1002/nbm.3062.

[44] Mikail Rubinov and Olaf Sporns. "Complex network measures of brain connectivity: uses and interpretations". In: Neuroimage 52.3 (2010), pp. 10591069. URL: https://doi.org/10.1016/j.neuroimage.2009.10.003.

[45] Benjamin Sinclair et al. "Heritability of the network architecture of intrinsic brain functional connectivity". In: NeuroImage 121 (2015), pp. 243-252. ISSN: 10538119. URL: https://doi.org/10.1016/j.neuroimage.2015.07.048.

[46] Aric A. Hagberg, Daniel A. Schult, and Pieter J. Swart. "Exploring Network Structure, Dynamics, and Function using NetworkX". In: Proceedings of the 7th Python in Science Conference. Ed. by Gaël Varoquaux, Travis Vaught, and Jarrod Millman. Pasadena, CA USA, 2008, pp. 11-15. URL: https://permalink.lanl. gov/object/tr?what=info:lanl-repo/lareport/LA-UR-08-05495.

[47] Eric Jones, Travis Oliphant, Pearu Peterson, et al. SciPy: Open source scientific tools for Python. 2001. URL: http://www.scipy.org/.

[48] Albert-László Barabási and Réka Albert. "Emergence of scaling in random networks". In: science 286.5439 (1999), pp. 509-512. URL: https ://doi.org/ 10.1126/science.286.5439.509.

[49] Paul Erdös, Alfréd Rényi, et al. "On random graphs". In: Publicationes mathematicae 6.26 (1959), pp. 290-297. 
[50] Gabor Csardi and Tamas Nepusz. "The igraph software package for complex network research". In: Inter Journal Complex Systems (2006), p. 1695. URL: http: //igraph.org.

[51] Kamalaker Dadi et al. "Benchmarking functional connectome-based predictive models for resting-state fMRI". In: NeuroImage 192 (2019), pp. 115-134. URL: https://doi.org/10.1016/j.neuroimage.2019.02.062.

[52] Xiao Liu et al. "The change of functional connectivity specificity in rats under various anesthesia levels and its neural origin". In: Brain topography 26.3 (2013), pp. 363-377. URL: https://doi.org/10.1007/s10548-012-0267-5.

[53] Joanes Grandjean et al. "Optimization of anesthesia protocol for resting-state fMRI in mice based on differential effects of anesthetics on functional connectivity patterns". In: NeuroImage 102.Part 2 (2014), pp. 838-847. ISSN: 1053-8119. URL: https://doi.org/10.1016/j.neuroimage.2014.08.043.

[54] Elisabeth Jonckers et al. "Different anesthesia regimes modulate the functional connectivity outcome in mice". In: Magn. Reson. Med. 72.4 (2014), pp. 11031112. URL: https://doi.org/10.1002/mrm.24990.

[55] Ruggero G Bettinardi et al. "Gradual emergence of spontaneous correlated brain activity during fading of general anesthesia in rats: Evidences from fMRI and local field potentials". In: NeuroImage 114 (2015), pp. 185-198. URL: https : //doi.org/10.1016/j.neuroimage.2015.03.037.

[56] Jaakko Paasonen et al. "Functional connectivity under six anesthesia protocols and the awake condition in rat brain". In: NeuroImage 172 (2018), pp. 9-20. URL: https://doi.org/10.1016/j.neuroimage.2018.01.014.

[57] Alex Fornito, Andrew Zalesky, and Michael Breakspear. "Graph analysis of the human connectome: promise, progress, and pitfalls". In: Neuroimage 80 (2013), pp. 426-444. URL: https://doi.org/10.1016/j.neuroimage.2013.04.087.

[58] Tingting Xu et al. "Network analysis of functional brain connectivity in borderline personality disorder using resting-state fMRI". In: NeuroImage: clinical 11 (2016), pp. 302-315. URL: https://doi.org/10.1016/j.nicl.2016.02.006.

[59] Demetrius Ribeiro de Paula et al. "A method for independent component graph analysis of resting-state fMRI". In: Brain and behavior 7.3 (2017), e00626. URL: https://doi.org/10.1002/brb3.626.

[60] Zhifeng Liang, Jean King, and Nanyin Zhang. "Intrinsic organization of the anesthetized brain". In: Journal of Neuroscience 32.30 (2012), pp. 10183-10191. URL: https ://doi .org/10.1523/JNEUROSCI .1020-12.2012.

[61] Aaron Alexander-Bloch et al. "The discovery of population differences in network community structure: new methods and applications to brain functional networks in schizophrenia". In: Neuroimage 59.4 (2012), pp. 3889-3900. URL: https : // doi.org/10.1016/j.neuroimage.2011.11.035. 
[62] Cedric E Ginestet, Arnaud P Fournel, and Andrew Simmons. "Statistical network analysis for functional MRI: summary networks and group comparisons". In: Frontiers in computational neuroscience 8 (2014), p. 51. URL: https://doi. org/10.3389/fncom.2014.00051.

[63] Kathleen A Williams et al. "Comparison of $\alpha$-chloralose, medetomidine and isoflurane anesthesia for functional connectivity mapping in the rat". In: Magn. Reson. Imaging 28.7 (2010), pp. 995-1003. URL: https://doi.org/10.1016/j. mri.2010.03.007.

[64] Elisabeth Jonckers et al. "Functional connectivity fMRI of the rodent brain: comparison of functional connectivity networks in rat and mouse". In: PLoS One 6.4 (2011), e18876. URL: https://doi.org/10.1371/journal.pone.0018876.

[65] Mark D Humphries and Kevin Gurney. "Network 'small-world-ness': a quantitative method for determining canonical network equivalence". In: PloS one 3.4 (2008), e0002051. URL: https://doi .org/10.1371/journal .pone.0002051. 


\section{Appendix A. Distribution of correlation coefficients between two random variables}

Distributions of the correlation coefficient of multivariate can be found in [33]. The probability density function $f$ of the correlation coefficient $r$ between two Gaussian processes with $N$ samples is then:

$$
f(r)=\frac{\Gamma\left(\frac{1}{2} N_{1}\right)}{\pi^{1 / 2} \Gamma\left(\frac{1}{2}\left(N_{1}-1\right)\right)}\left(1-r^{2}\right)^{\left(N_{1}-3\right) / 2} \quad(-1<r<1)
$$

with $N_{1}=N-1$. The hypothesis test for the correlation coefficient advised in [33] is then:

$$
\left(N_{1}-1\right)^{1 / 2} \frac{|r|}{\left(1-r^{2}\right)^{1 / 2}}>t_{N_{1}-1}^{*}(\alpha)
$$

with $t_{N_{1}-1}^{*}(\alpha)$ the two-tailed $100 \alpha \%$ point of the $t_{N_{1}-1}$ distribution. In practice, for $N>4$, the Fisher's $z$ transform $z=\tanh ^{-1} r=\frac{1}{2} \log \frac{1+r}{1-r}$ is applied. For uncorrelated processes, the expected correlation is centered around 0 and the distribution follows a normal distribution $\mathcal{N}\left(0, \frac{1}{\left(N_{1}-2\right)}\right)$. This is why the confidence interval given in 31 for wavelet correlations and presented in Eq,1 is proposed here.

\section{Appendix B. Distributions of correlation coefficients with or without samples corrupted by movement}

In order to evaluate the effects of removing data corrupted by movement artefacts, distributions of correlations with or without applying the scrubbing method on samples are proposed in Fig, B1. Few rats show distributions corrupted by movement, except for Iso-L Med-L and Ure-L. The correction of movements (scrubbing) enables to homogenize the distributions of correlation values. Samples associated with movements lead to high values of correlations.

\section{Appendix C. Circular graphs with significant connections}

Circular graphs with significant correlation values obtained on rats are given in Fig. C1.

\section{Appendix D. Other projections and measures}

The measure of closeness centrality (clo) is also proposed in this section [44. It is defined as:

$$
\operatorname{clo}_{i}=\frac{n_{r i}-1}{\sum_{j \in \mathcal{G}_{r i}} d(i, j)},
$$

where $j$ is in $\mathcal{G}_{r i}$ the set of all nodes reachable from $i, d(i, j)$ is the distance of the shortest path between $(i, j)$, and $n_{r i}$ is the number of reachable nodes from $i$. 

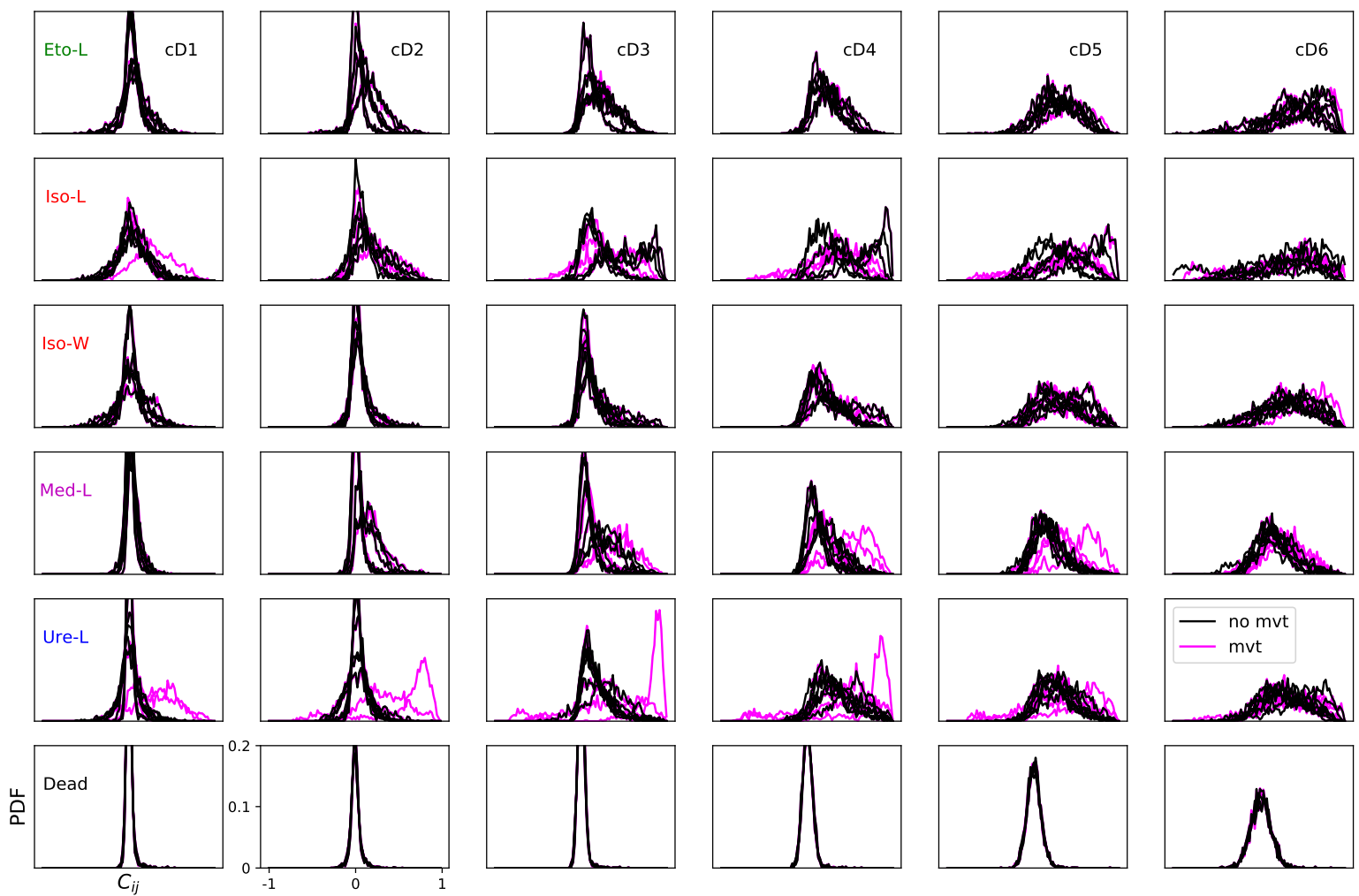

Figure B1. Distributions of correlations with or without removal of samples corrupted by movements (with and without movement, in magenta and black, respectively). Figures are given in row by group and in column by wavelet scale. Individual curves for each rat are plotted, with movement, i.e. taking all samples, in background, and no movement, using scrubbing, in foreground. Histograms are computed using 100 bins between -1 and 1 .
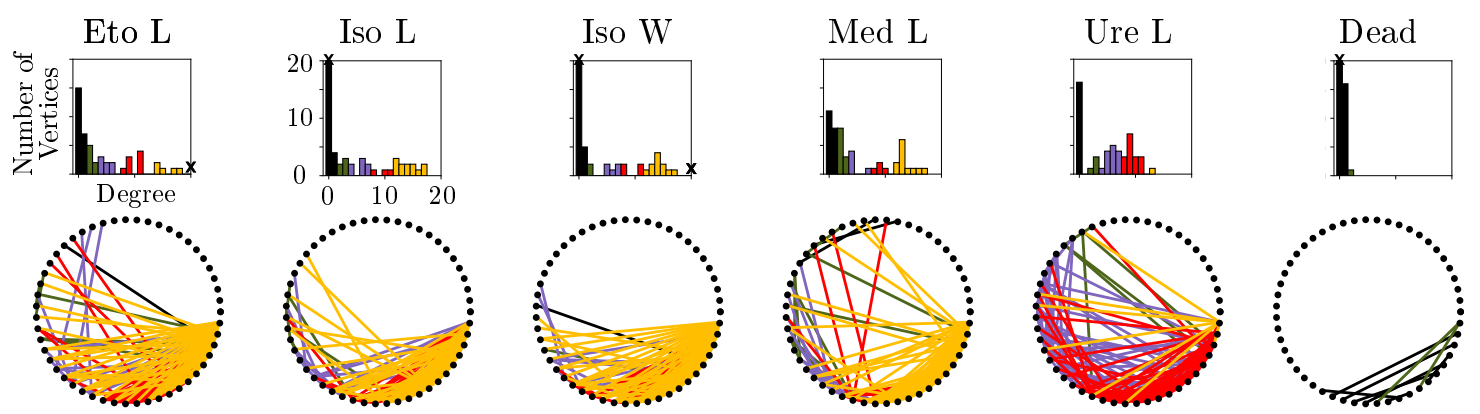

Figure C1. Circular graphs obtained with significant values. Average matrices are computed with significant values from individual rats then thresholded at a maximal value of $\delta=0.1$. 


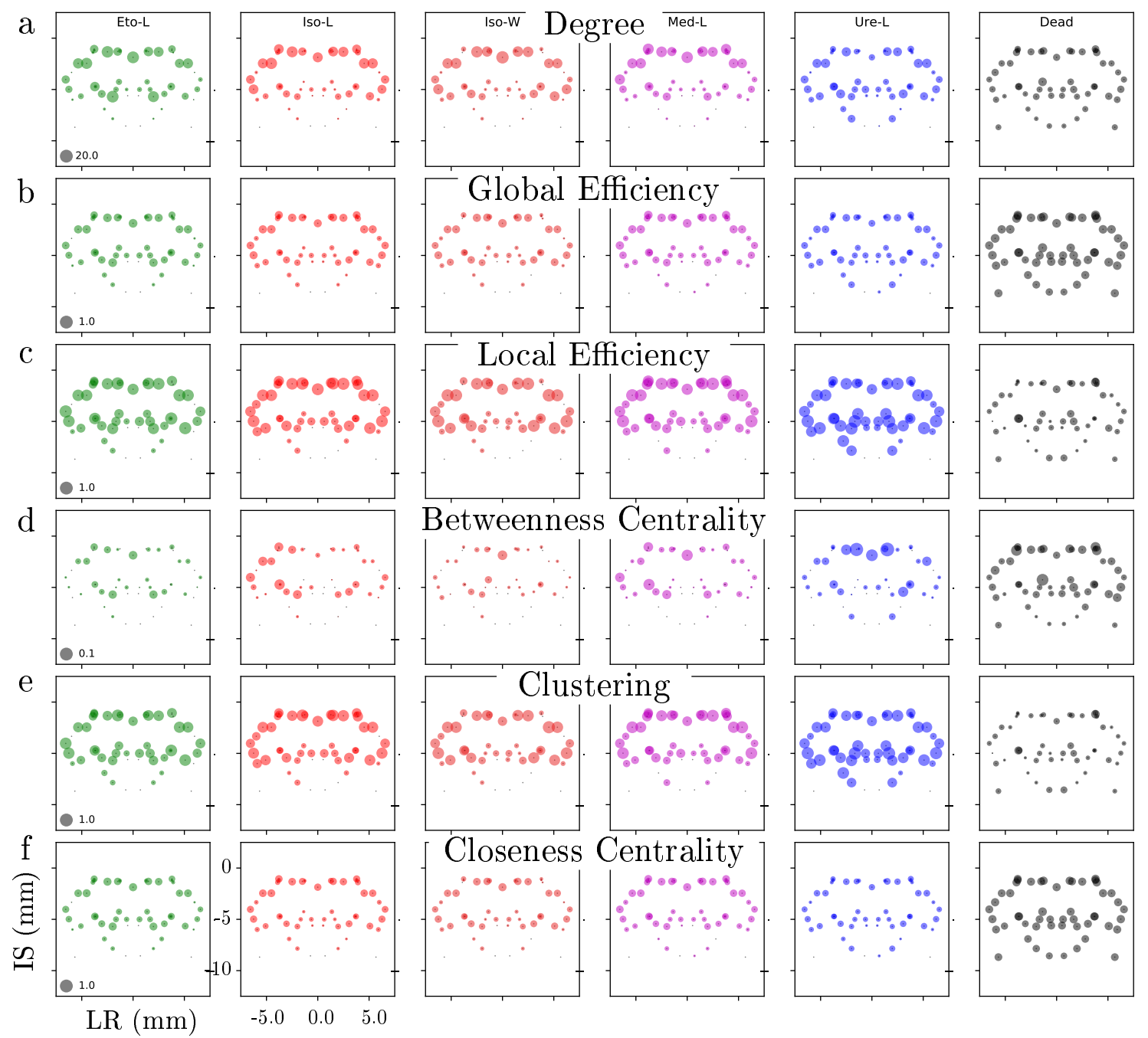

Figure D1. Graph measures in LR-IS with all FC. Average measures with all correlation coefficients even those not significant.

Representations of the different node measures obtained from graphs constructed on non thresholded correlations matrices, i.e. without removing non significant values are proposed in Fig. D1. Graphs are obtained at a density of 0.1. Areas names and position of the centers of gravity in the three projections are shown in Fig. D4.

The effect of not removing significant edges shows uniform measures on the Dead group. This group could serve as reference with patterns comparable to those obtained from random networks. Local efficiency and clustering provide similar information. Global efficiency and closeness centrality provide similar information. 


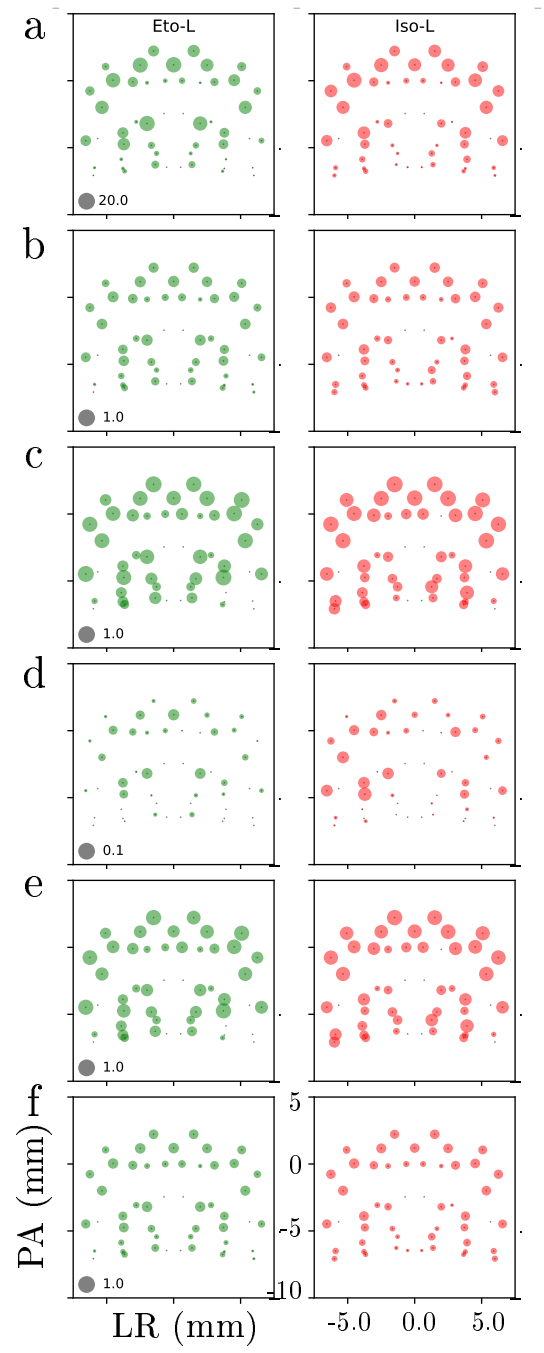

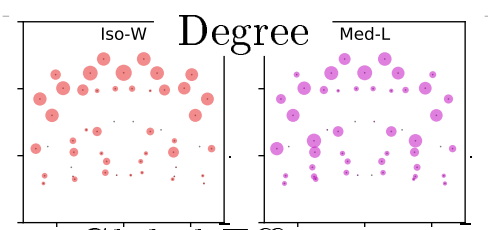
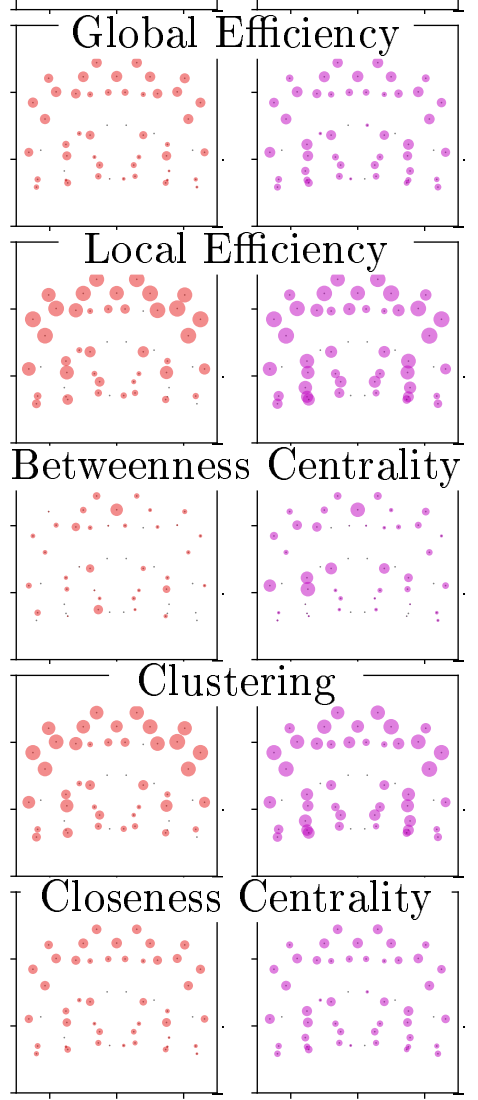

Centrality

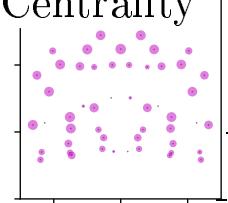

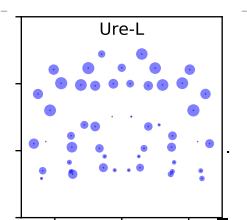
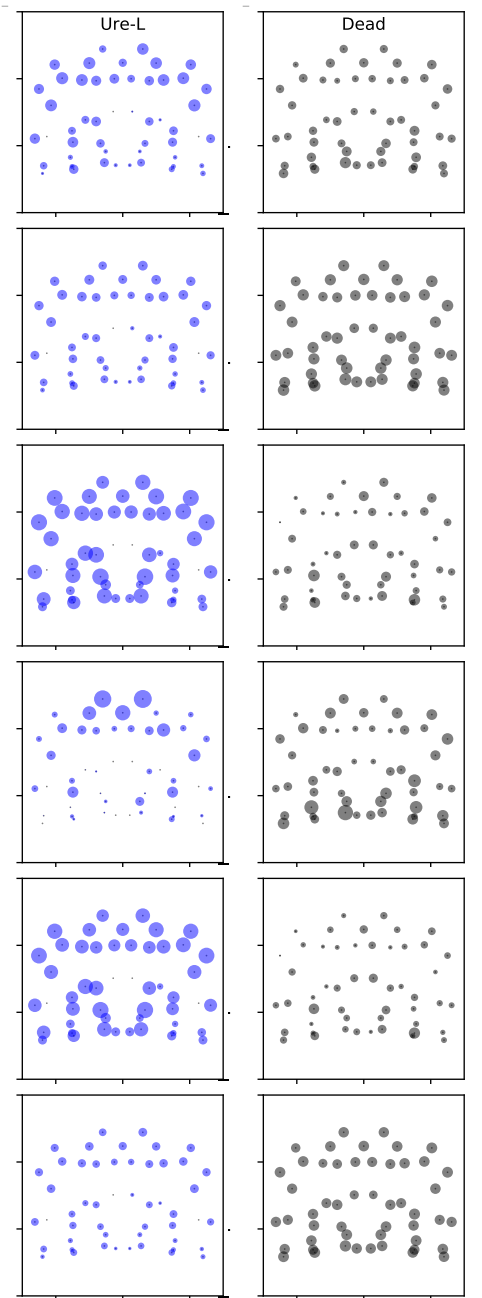

Figure D2. Graph measures in LR-AP with all FC. Average measures with significant correlation coefficients even those not significant. 

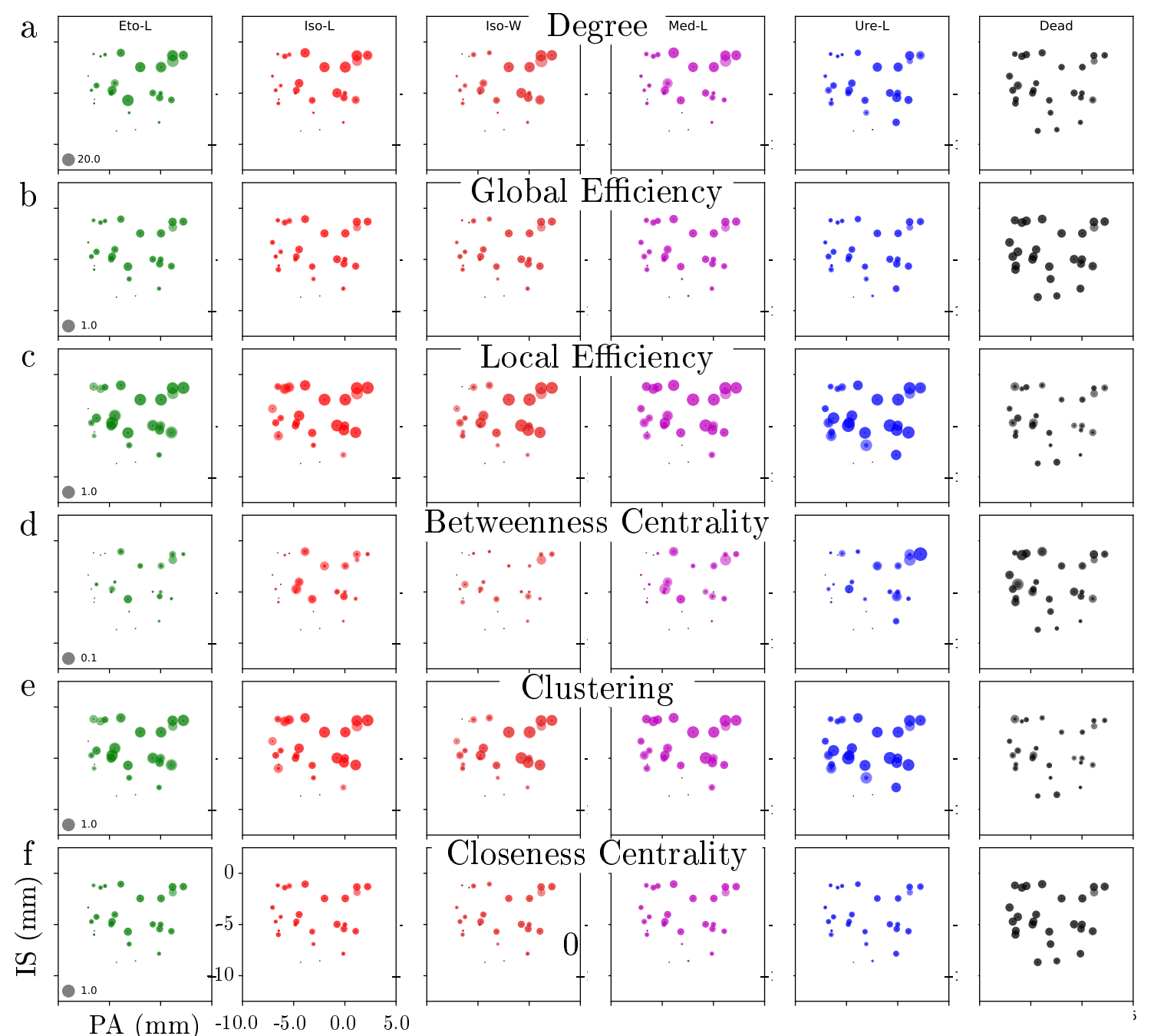

PA (mm) $\quad-10.0 \quad-5.0 \quad 0.0 \quad 5.0$

Figure D3. Graph measures in PA-IS with all FC. Average measures with significant correlation coefficients even those not significant. 

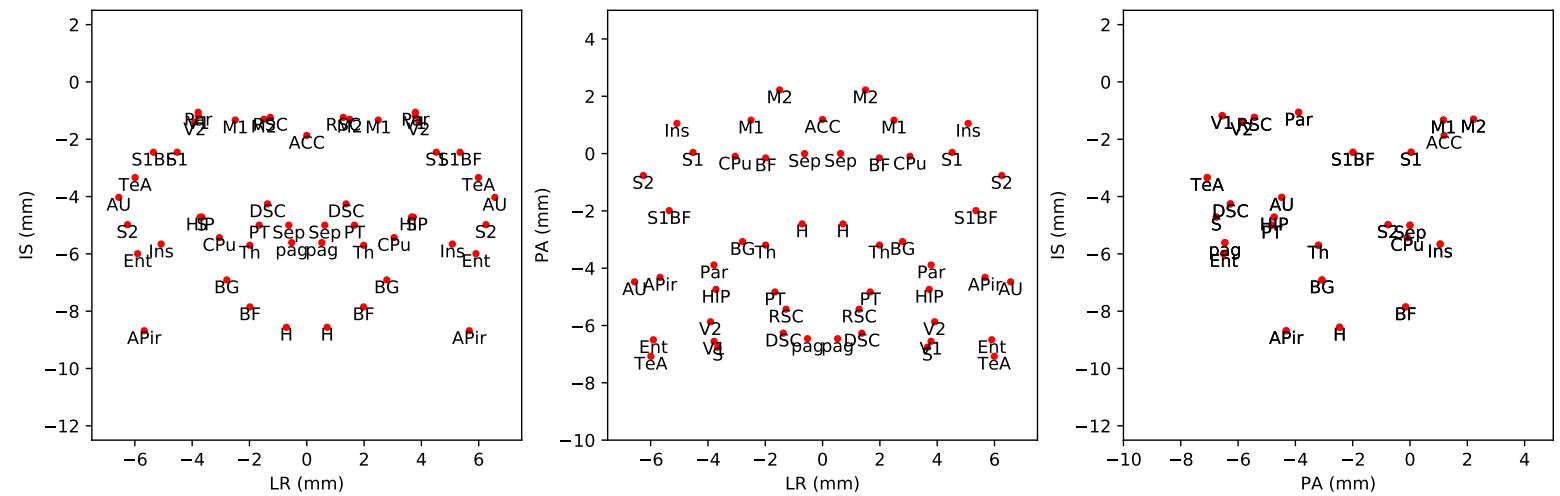

Figure D4. Area positions and names in different projections. List of areas and abbreviations - l: left, r: right, ACC: anterior cingulate cortex, APir: amygdalopiriform transition area, AU: auditory cortex, BF: basal forebrain region, BG: basal ganglia, CPu: caudate-putamen striatum, DSC: superior colliculus, Ent: entorhinal area, Ins: insular cortex H: hypothalamic region, HIP: hippocampus, M1: primary motor cortex, M2: supplementary motor cortex, pag: periaqueductal gray, Par: parietal association cortex, PT: pretectal region, RSC: retrosplenial cortex, S: subiculum S1: somatosensory 1, S1BF: somatosensory 1 barrel field, S2: somatosensory 2, Sep: septal region, TeA: temporal cortex association area, Th: thalamus, V1: primary visual cortex, V2: secondary visual cortex. 